\title{
An Image Processing Based Fungus Detection System For Mangoes
}

\author{
Ananthi N, Akshaya S, Aarthi B, Aishvarya J, Kumaran K
}

\begin{abstract}
Fruits which grow with high yield in many states of India are rich in proteins. But due to addition of excess pesticides and chemicals intake of these fruits lead to serious health problems. It is necessary to identify the presence of chemical in the fruits before consuming it. In this project we have planned to develop an image processing technique to analyze whether the fruit is free from chemicals and fungus. In our paper, we have implemented MATLAB used as well as fungus present in the fruit. We capture the images of the fruit or we use datasets and train the database with different color-based changes that happen after adding chemicals to the fruit. The enhancement process is carried out in the captured image. Then image is segmented to hit the regions with affected spots in the fruit. K-means method is used to carry out the segmentation process. The input image is compared with the given data set for training to identify the images. In this way unhealthy fruits can be identified and the affected spots in the fruit can be detected.

Keywords: MATLAB; k-mean; image processing; segmentation.
\end{abstract}

\section{INTRODUCTION}

Diseases in fruits cause health problems to humans and leads to loss in production of healthy fruits in the agricultural sector. It is essential to monitor and detect the presence of chemicals and diseases in fruits. These diseases that are detected in fruits reduce the quality of the yield. Detecting the diseases and chemically affected spots in fruits at an early stage can lead to a healthy consumption. Diseases are visible as spots in fruits and if not detected can lead to severe losses. The use of pesticides is a reason for the diseased fruit. The diseases can be mealybugs, blackspots, bactrocera or antracnose. Mealybugs are like insects that appear in fruits of the plants in the form of disease. Black spot is a form of fungal disease appearing in fruits that cause reduction in quality and quantity of the fruits.

Revised Manuscript Received on November 30, 2019.

* Correspondence Author

Dr. Ananthi N*, Department of Information Technology, Easwari Engineering College, Chennai, India, nandhura@gmail.com

Akshaya S, Department of Information Technology, Easwari Engineering College, Chennai, India akshayasahas3004@gmail.com

Aarthi B, Department of Information Technology, Easwari Engineering College, Chennai, India, aarthi.rt.1212@gmail.com

Aishvarya J, Department of Information Technology, Easwari Engineering College, Chennai, India, aishwonder@gmail.com

Kumaran K, Department of Information Technology, Easwari

(C) The Authors. Published by Blue Eyes Intelligence Engineering and Sciences Publication (BEIESP). This is an open access article under the CC-BY-NC-ND license http://creativecommons.org/licenses/by-nc-nd/4.0/ Engineering College, Chennai, India,_kumaran.me.cse@gmail.com

Anthracnose can cause severe damage to the fruit and can affect the quality of fruit. Bactrocera is a kind of fruit fly that affects major portions of the fruit. It becomes unhealthy for consumption. So, there is a need to identify and detect the chemicals and fungus spots in fruits using image processing method. It is carried out for visualization, image sharpening and restoration, image retrieval, measurement of pattern and image recognition. So early detection of fruit diseases can lead to proper intake fruits.

\section{RELATED WORKS}

One of the globally consumed fruit is banana. It has $16 \%$ in total world's fruit production. The ripening stages of banana have been determined using neural network and deep learning [1]. Mango is a season fruit. It is one of the favorite tropical fruit. The method for classification of mango by its size and shape is developed. In this work they automated the sorting of mango by digital image analysis and MATLAB toolbox [4]. A computer vision-based apple grading is developed. It has totally 6 steps. In this they separate the defected area using Multi-Layer Perceptron (MLP) neural networks. For grading they compare the performance of MLP, Support Vector Machine (SVM) and K-Nearest Neighbor (KNN) [2]. Diagnosis of pomegranate plant defects had been developed using neural networks. In this work they use K-means clustering segmentation and diagnose the diseases like fruit rot, fruit spot and bacterial blight [8].

\section{SYSTEM DESIGN}

In our proposed system, fruits with defects will be identified using image processing technique. The chemically affected portions of the fruits are also identified. The input fruit image is given in the form of RGB image. The preprocessing technique has been applied to enhance the quality of image. The segmentation process is carried out to highlight the required portion of the fruit. Feature extraction is carried out using SFTA algorithm and classified based on data set. The data set has been trained using DNN algorithm.

Published By:

Blue Eyes Intelligence Engineering 


\section{An Image Processing based fungus detection system for mangoes}

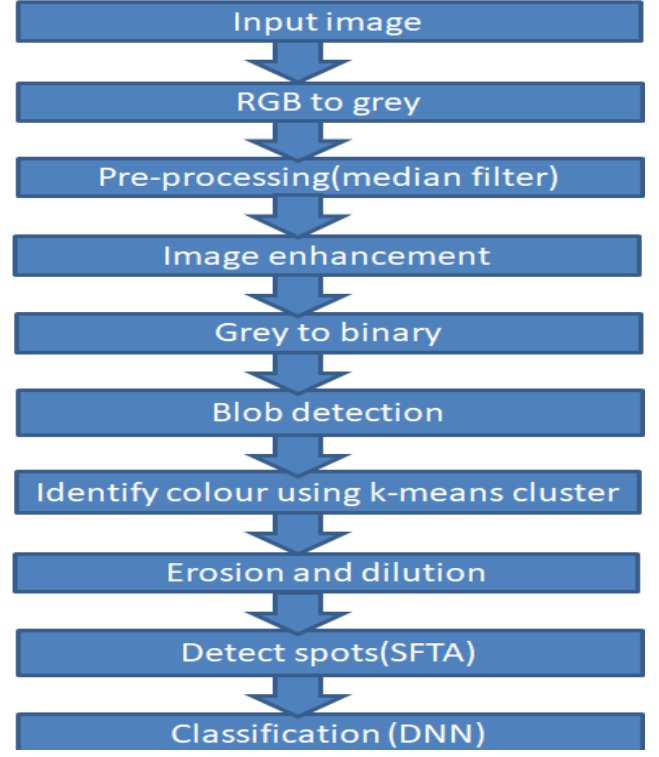

Figure 1. Flow diagram.

\section{A. Pre-processing}

The fruit for which defect is to be found is captured and considered as the input. The input colour image is processed to grey scale image. Pre-processing is carried out using median filter. It is a non-linear digital filtering technique. This stage is very important to produce better quality and accurate result. Median filter is used to remove noises such as gaussian noise, salt and pepper noise.

\section{B. Blob Detection}

After completion of pre-processing stage, the enhanced method is applied in binary image. In blob detection, the regions that differ in properties such as size, colour are compared with the surrounding regions and highlighted. The centroid is then calculated with the help of $\mathrm{x}$ and $\mathrm{y}$ coordinates values of the region.

\section{K-means segmentation algorithm}

K-means is the simplest unsupervised learning algorithms used to solve the standard agglomeration drawback. The procedure follows an easy and simple ways to classify given information set through a definite range of clusters (assume $\mathrm{k}$ clusters). The main idea is to outline $\mathrm{k}$ centroids, one for every cluster.

Algorithm for K Means Algorithm

1. Get the input image.

2. Get the clusters to be formed number.

3. Transform RGB image as gray image.

4. Modify the 2-D image into 1-D array of length " $r \times c$ ".

5. Calculate the intensity range.

Range $=[($ Maximum value $)$ - (Minimum value)]

6. Calculate the centroid value Centroid 1 = Range/Number of clusters Centroid $2=(2 \times$ Centroid 1$)$

7. Calculate the difference between the first intensity value and the other centroid values. image is converted into binary format. Blob detection

8. Group the intensity values into the corresponding clusters, based on minimum value

9. Repeat $1 \& 2$ for all the other intensity values of the image.

$$
\text { Centroid } 3=(3 \times \text { Centroid } 1)
$$$$
\text { Centroid } \mathrm{n}=(\mathrm{n} \times \text { Centroid } 1)
$$

D. Watershed Algorithm

In this step, the segmented image is processed to remove unwanted pixels from the segmented region. It is a transformation done on the grey scale image, Erosion and dilution processes are carried out. This algorithm is used in image processing for segmentation.

\section{E. Feature Extraction}

In this process, texture analysis is carried out to find the texture boundaries of the regions in the fruit. The variations in the intensity values such as roughness or smoothness are quantified. It is described as a variation in the pixel intensities. In texture analysis, the image is filtered, and texture of the image is characterised to provide information of values of intensity of the pixels in the image. If the texture is smooth, the values around a pixel will be small else for rough texture the values would be larger. In our system we have used SFTA technique for feature extraction. Around six fixtures namely colour, texture, size, energy, shape and contrast will be extracted from the fruit image after segmentation process. Each feature will be set to a threshold value. Based on the threshold values, the segmented image has been undergone any three-feature extraction process. The feature matrix is show in figure 2.

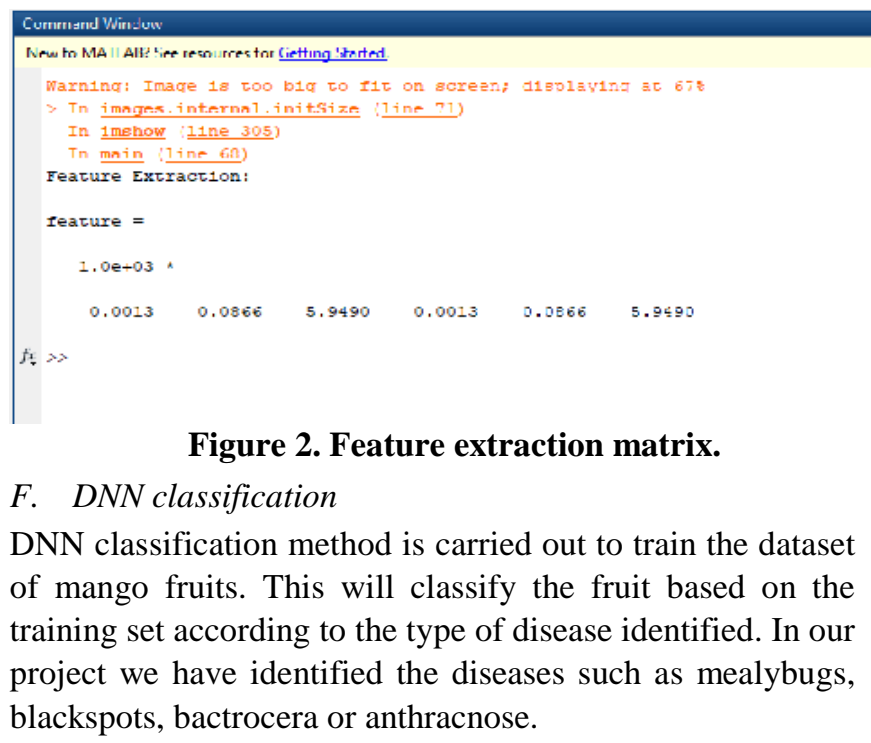

\section{EXPERIMENTAL RESULTS}

The original fruit image is first pre-processed to remove unwanted noises to achieve better quality of image and accurate result. Pre-processed image is transformed from RGB to binary. The blob detection is processed to extract the fruit region. Segmentation is carried out to highlight the defected portions in the fruit. Finally, the features have been extracted to identify whether the fruit is affected or not. 


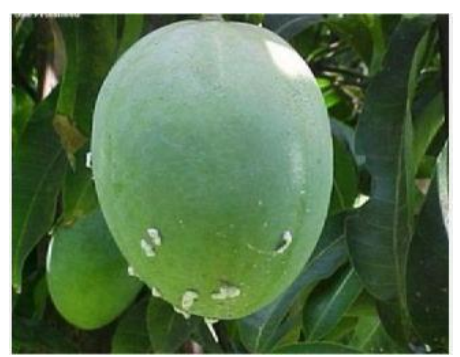

(a)

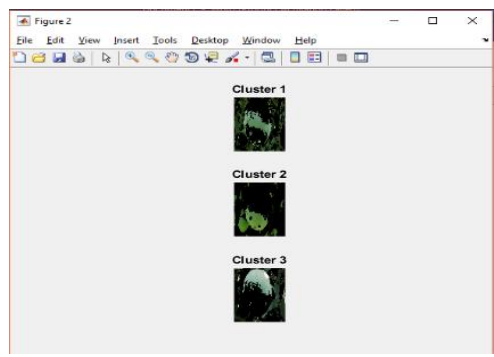

(b)

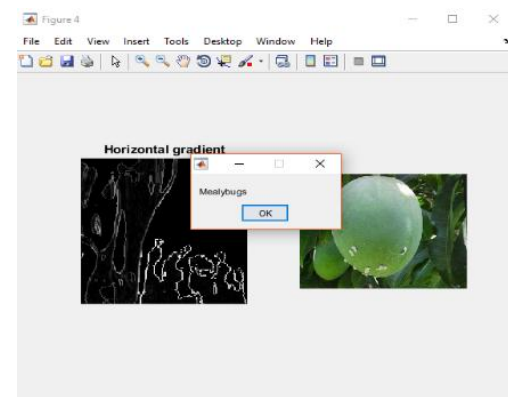

(c)

Figure 3. Mealybugs (a) Input image, (b) cluster, (c) output screen

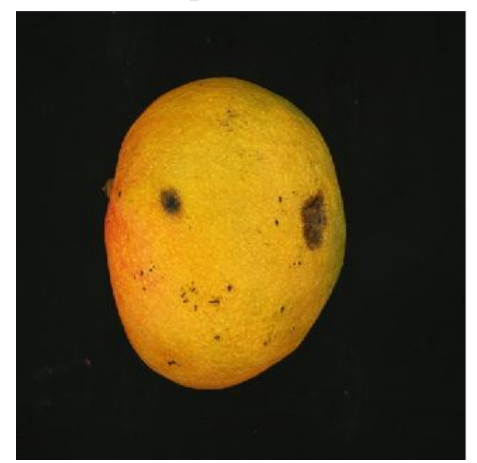

(a)

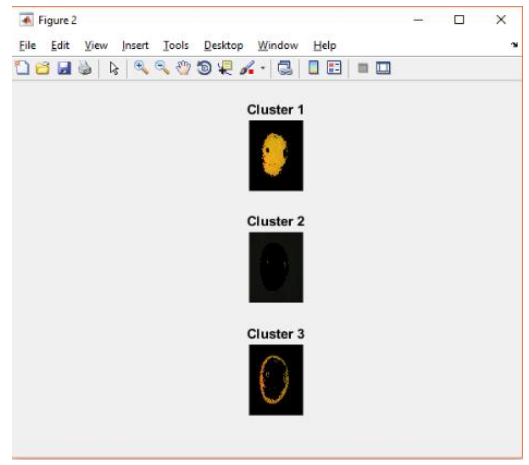

(b)

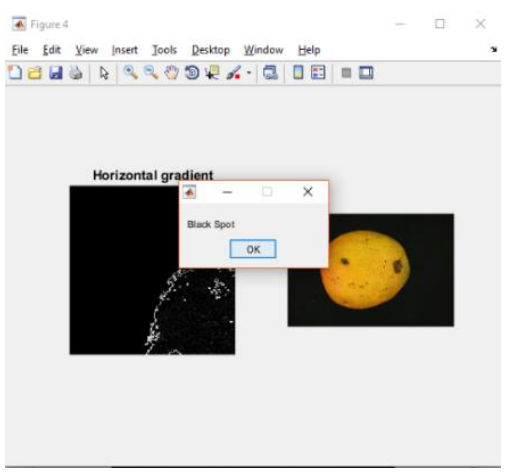

(c)

Figure 4. Black spots (a) Input image, (b) cluster, (c) output screen

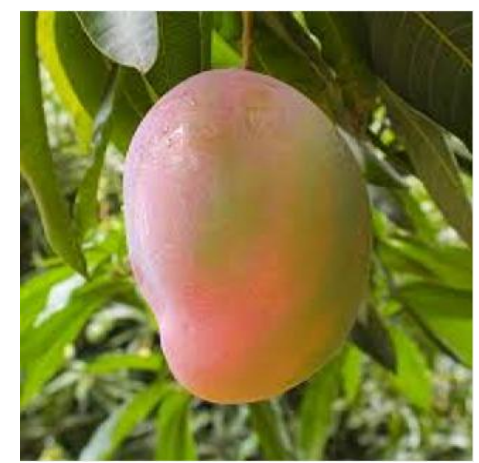

(a)

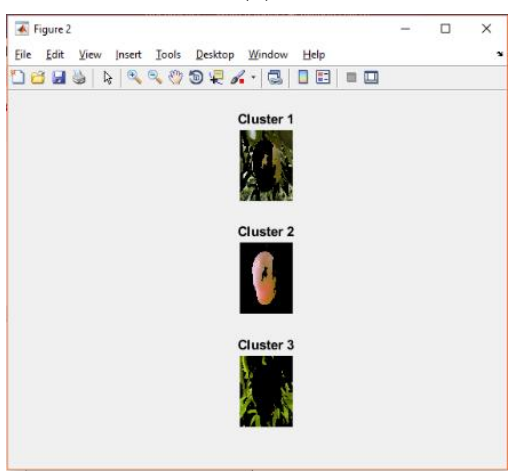

(b)

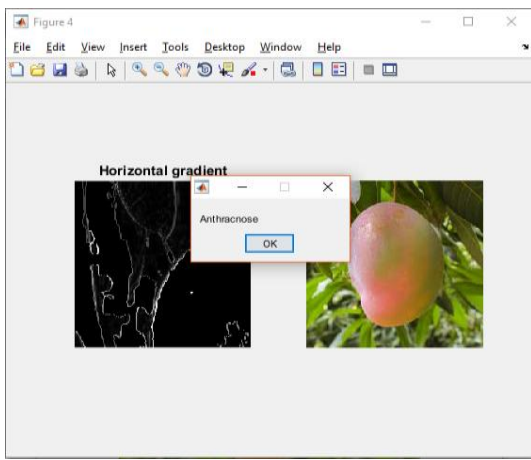

(c)

Figure 5. Anthracnose (a) Input image, (b) cluster, (c) output screen 


\section{An Image Processing based fungus detection system for mangoes}

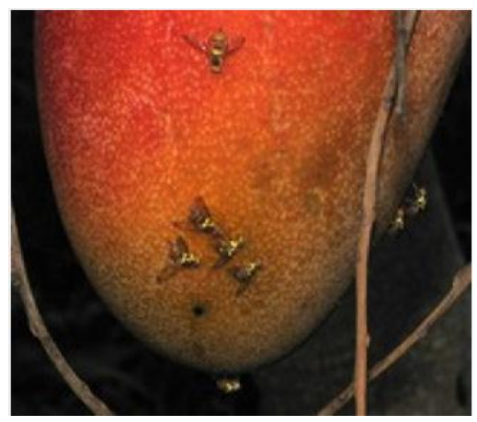

(a)

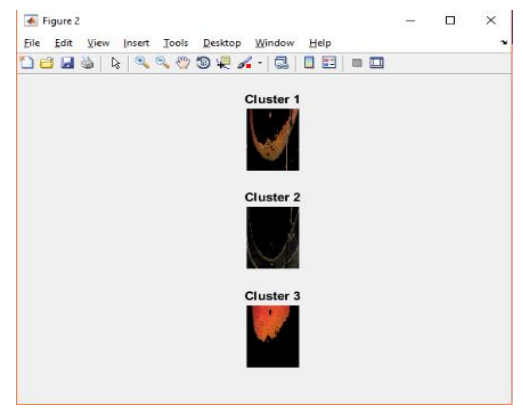

(b)

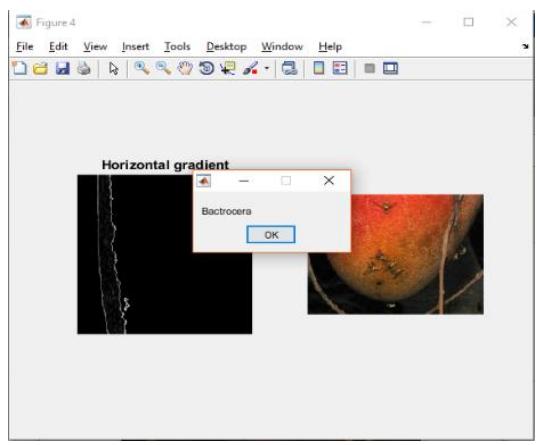

(c)

Figure 6. Bactrocera (a) Input image, (b) cluster, (c) output screen

Performance analysis of the training data set using neural networks is evaluated as shown in figure 7 and figure 8.

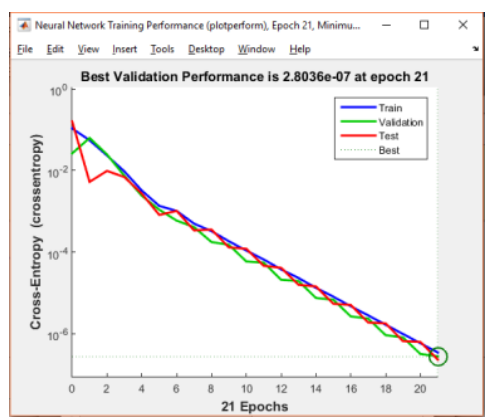

Figure 7.

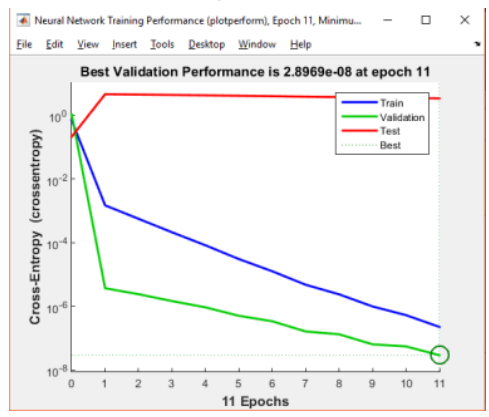

Figure 8.
The performance of the testing image will be based on the number of trained images. In the figure 7 the process has taken 21 iterations to complete. This is because the trained images are less in number. On comparison, in figure 8 the process has taken only 11 iterations to complete as more number of images are trained. It takes few milli seconds to complete the process. As the number of iterations decreases the time taken to complete the process also reduces. Thus, performance can be increased by training more data.

\section{CONCLUSION}

In our system image processing technique was used to identify the defects in mango fruit. The affected portions of the fruit were identified. Through this process the type of defect present in the fruit were also identified. Based on the results, it can be concluded that DNN classification is a key step to produce reliable outcome in detecting the presence of diseases in mango fruit. In future we plan to develop a generic system for fruits.

\section{ACKNOWLEDGEMENT}

This work was supported by DST-FIST Programme No.SR/FST/College-110/2017, Government of India

\section{REFERENCES}

1. Yan Zhang, Jian Lian, Mingqu Fan and YuanjieZheng,"Deep indicator for fine-grained classification of banana's ripening stages", Zhang et al. EURASIP Journal on Image and Video Processing (2018) 2018:46 https://doi.org/10.1186/s13640-018-0284-8.

2. PaymanMoallem, Alireza Hossein Pourghassem,"Computer vision-based apple grading for golden delicious apples based on surface features", INFORMATION PROCESSING IN AGRICULTURE XXX (2016) XXX-XXX.

3. Siddharth Singh Chouhan, Ajay Kaul1, Uday Pratap Singh and Sanjeev Jain,"Bacterial foraging optimization based Radial Basis Function Neural Network (BRBFNN) for identification and classification of plant leaf diseases: An automatic approach towards Plant Pathology", 2169-3536 (C) 2017 IEEE.

4. DameshwariSahu, ChiteshDewangan, "Identification and Classification of Mango Fruits Using Image Processing", International Journal of Scientific Research in Computer Science, Engineering and Information Technology@ 2016 IJSRCSEIT Volume 2 | Issue 2 | ISSN: 2456-3307.

5. Sandesh Raut, Amit Fulsunge,"Plant Disease Detection in Image Processing Using MATLAB", DOI:10.15680/IJIRSET.2017.0606034.

6. AmelH.Abbas, MarwaA.Shamel,"Identify and Classify Normal and Defects of Prunus_armeniaca Using Imaging Techniques", Kurdistan Journal of Applied Research (KJAR) | Print-ISSN: 2411-7684 Electronic-ISSN: 2411-7706 | kjar.spu.edu.iq

7. Volume 2 | Issue 3 | August 2017 | DOI: 10.24017/science.2017.3.11.

8. Tejal Deshpande, Sharmila Sengupta, K.S.Raghuvanshi,"Grading\& Identification of Disease in Pomegranate Leaf and Fruit", Teja Deshpande et al, / (IJCSIT) International Journal of Computer Science and Information Technologies, Vol. 5 (3) , 2014, 4638-4645

9. www.ijcsit.

10. MrunmayeeDhakate, Ingole A. B.,"Diagnosis of Pomegranate Plant Diseases using Neural Network",978-1-4673-8564-0/15/\$31.00 (C) 2015 IEEE.

11. S. R. Dubey and A. S. Jalal, "Application of Image Processing in Fruit and Vegetable Analysis: A Review," J. Intell. Syst., vol. 24, no. 4, pp. 405-424, 2015. 


\section{AUTHORS PROFILE}

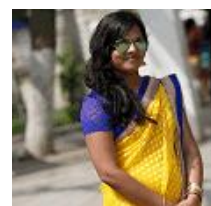

N.Ananthi received her M.E. degree from Manonmanium Sundaranar University, B.E. degree from Bharathidasan University in the year 2002 and 2000 respectively. She received her Ph.D degree in the year 2016 from Anna University. She is SAP NETWEAVER-ABAP certified consultant in ECC 5.0 She is the recipient of Mission 10X certification "In Pursuit of Excellence in Engineering Education through Innovation". She has received funds from AICTE, TNSCST, IET for student projects and to conduct of Workshop, Seminar and Faculty Development Program. She has 18 years of teaching experience and she has published more than 16 research papers in international journals and conferences. Her current research interests include Wireless Networks and IoT.

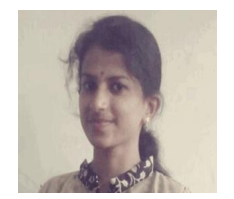

Akshaya S studying B.Tech Degree in Information Technology under Anna University, Tamilnadu, India in Her current research interests include Network Security and Cloud Computing.

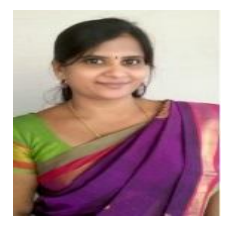

Aarthi B studying B.Tech Degree in Information Technology under Anna University, Tamilnadu, India in Her current research interests include Network Security and Cloud Computing.

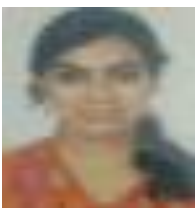

Aishvarya J studying B.Tech Degree in Information Technology under Anna University, Tamilnadu, India in Her current research interests include Network Security and Cloud Computing.

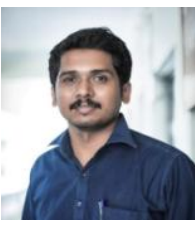

K.Kumaran received his M.E Degree in Computer Science and Engineering from Anna University and B.Tech Degree in Information Technology from Anna University, Tamilnadu, India in 2013 and 2009 respectively. He has 7.9 years of teaching experience including experience in IT Industry. He has more than 5 research papers in international journals and conferences. His current research interests include Network Security and Image Processing. 